\title{
Mandenkan
}

MANDENIKAN Bulletin semestriel d'études linguistiques mandé

51 | 2014

Grammatical Sketch of Beng

\section{List of Notation and Glosses}

\section{Denis Paperno}

\section{(2) OpenEdition}

Journals

Electronic version

URL: https://journals.openedition.org/mandenkan/548

DOI: 10.4000/mandenkan.548

ISSN: 2104-371X

\section{Publisher}

Llacan UMR 8135 CNRS/Inalco

\section{Printed version}

Date of publication: 1 June 2014

Number of pages: 125-126

ISSN: 0752-5443

\section{Electronic reference}

Denis Paperno, "List of Notation and Glosses", Mandenkan [Online], 51 | 2014, Online since 01 June 2014, connection on 08 July 2021. URL: http://journals.openedition.org/mandenkan/548; DOI: https:// doi.org/10.4000/mandenkan.548

This text was automatically generated on 8 July 2021.

Les contenus de Mandenkan sont mis à disposition selon les termes de la Licence Creative Commons Attribution - Pas d'Utilisation Commerciale - Partage dans les Mêmes Conditions 4.0 International. 


\section{List of Notation and Glosses}

\section{Denis Paperno}

\begin{tabular}{|l|l|}
\hline & high tone \\
\hline & low tone \\
\hline & mid tone \\
\hline & falling (high to low) tone \\
\hline & rising (low to high) tone \\
\hline & mid-high rising tone \\
\hline+ & mid-low falling tone \\
\hline$(\mathrm{x})$ & X is optional \\
\hline$*(\mathrm{x})$ & $\mathrm{X}$ is obligatory \\
\hline $\mathrm{X} / / \mathrm{Y}$ & $\mathrm{X}$ and $\mathrm{Y}$ are free variants \\
\hline+ & affirmative polarity series \\
\hline- & negative polarity series \\
\hline 1 & $1^{\text {st }}$ person \\
\hline 2 & $2^{\text {nd }}$ person \\
\hline 3 & $3^{\text {rd }}$ person or portemanteau series contracted with a 3sG non-subject pronoun \\
\hline
\end{tabular}




\begin{tabular}{|c|c|}
\hline AG & agent nominalization \\
\hline APUD & localization APUD ‘around' \\
\hline ATR & attributive marker \\
\hline BENEF & benefactive postposition \\
\hline BSQ & base form of the verb (often omitted from the gloss) \\
\hline $\mathrm{L}$ & low tone form of the verb \\
\hline CND & conditional series \\
\hline CNS & syntactic marker of a construction \\
\hline CONT & localization CONT 'in contact with' \\
\hline COP & copula verb \\
\hline DEF & definite article \\
\hline DISTR & distributive interpretation \\
\hline DT & temporal shift \\
\hline EMPH & emphatic particle \\
\hline EX & existential series \\
\hline FOC & focus pronoun series or focus construction marker \\
\hline НАВ & habitual series \\
\hline IN & localization IN ‘in’ \\
\hline ITER & iterativity \\
\hline MEN & means nominalization \\
\hline NEG & negation \\
\hline NGO & cancelled result or irreal conditional marker \\
\hline NMLZ & (event) nominalization \\
\hline $\mathrm{P}$ & postposition \\
\hline POS & substantivized possessive series, also used as a contrastive topic marker \\
\hline POST & localization POST 'behind' \\
\hline POSS & localization POSS 'in the possession' \\
\hline
\end{tabular}




\begin{tabular}{|l|l|}
\hline PST & preterite series \\
\hline PRF & perfect verb aspect \\
\hline PL & plural \\
\hline PLC & locative nominalization \\
\hline PROG & progressive form \\
\hline RES & resultative/stative form \\
\hline RFL & reflexive series \\
\hline SG & singular \\
\hline ST & stative series \\
\hline SUB & localization SUB 'under' \\
\hline SUPER & localization SUPER 'on, over' \\
\hline TOP & topic marker \\
\hline V & verb stem or verb within an idiomatic expression \\
\hline
\end{tabular}

\section{AUTHOR}

\section{DENIS PAPERNO}

University of Trento, Italy

denis.paperno@gmail.com 